\title{
Unresectable hepatoblastoma: current perspectives
}

\author{
This article was published in the following Dove Press journal: \\ Hepatic Medicine: Evidence and Research \\ I February 2017 \\ Number of times this article has been viewed
}

\author{
Angela D Trobaugh-Lotrario' \\ Rebecka L Meyers ${ }^{2}$ \\ Allison F O'Neill ${ }^{3}$ \\ James H Feusner ${ }^{4}$ \\ 'Department of Pediatric Hematology \\ and Oncology, Providence Sacred \\ Heart Children's Hospital, Spokane, \\ WA, ${ }^{2}$ Primary Children's Hospital, \\ University of Utah, Salt Lake City, \\ UT, ${ }^{3}$ Dana-Farber Cancer Institute \\ and Harvard University, Boston, MA, \\ ${ }^{4}$ Children's Hospital \& Research \\ Center Oakland, Oakland, CA, USA
}

Correspondence: Angela D TrobaughLotrario

Department of Pediatric Hematology and Oncology, Providence Sacred Heart Children's Hospital, I0I West 8th Avenue, Spokane, WA 99204, USA

$\mathrm{Tel}+\mathrm{I} 5094742777$

Fax +I 5094746222

Email angela.trobaugh@providence.org

\begin{abstract}
Although rare, hepatoblastoma is the most common pediatric liver tumor. Complete resection is a critical component for cure; however, most patients will have tumors that are not resected at diagnosis. For these patients, administration of neoadjuvant chemotherapy renders tumors resectable in most patients. For patients whose tumors remain unresectable after chemotherapy, liver transplantation is indicated (in the absence of active unresectable metastatic disease). In patients whose tumors remain unresectable after conventional chemotherapy, interventional techniques may serve as a promising option to reduce tumor size, decrease systemic toxicity, decrease need for liver transplantation, and increase feasibility of tumor resection.

Keywords: hepatoblastoma, unresectable, pediatric
\end{abstract}

\section{Introduction}

Although rare, hepatoblastoma (HB) is the most common pediatric liver tumor with at least 100 cases per year in the US and an increasing incidence. ${ }^{1-4}$ Patients with HB typically present at $<5$ years of age, with an increased risk seen in patients with low birthweight, maternal tobacco exposure, familial adenomatous polyposis coli, and Beckwith-Wiedemann Syndrome..$^{5-8}$

Complete resection is a critical component for cure; however, $60 \%-80 \%$ of patients' tumors are unresectable at diagnosis depending upon the surgical guidelines used. ${ }^{9,10}$ The surgery-based Evans staging system, previously used in America, was based on an attempted resection at diagnosis in all patients. It classified tumors as stage I (upfront resection with negative microscopic margins), stage II (upfront resection with positive microscopic margins), stage III (unresectable at diagnosis without metastatic disease), and stage IV (metastatic disease). In the intergroup study INT0098 (1989-1992), 32\% of patients were presented with stage I or II disease and $68 \%$ of patients were presented with stage III or IV tumors. ${ }^{9}$ In Europe, the International Childhood Liver Tumors Strategy Group (SIOPEL) introduced a radiology-based staging system called PRETreatment EXTent of disease (PRETEXT).${ }^{11}$ The PRETEXT system classifies tumors into one of four groups based upon the number of tumor free liver sections. PRETEXT I tumors have three adjoining sectors free of tumor, PRETEXT II tumors have two adjoining sectors free of tumor, PRETEXT III tumors have one sector free of tumor, and PRETEXT IV tumors have no sectors free of tumor. In the most recent high risk (HR) SIOPEL trial SIOPEL 4 (which included patients with metastatic disease, PRETEXT IV tumors, extrahepatic abdominal disease, vascular 
invasion, and/or low alpha fetoprotein), the patients had 3\%, $27 \%, 44 \%$, and 26\% PRETEXT I, II, III, and IV tumors, respectively. ${ }^{12}$ In the most recent standard risk (SR) SIOPEL trial SIOPEL 3SR (which included patients without HR features), the patients had $8 \%, 52 \%$, and $40 \%$ PRETEXT I, II, and III tumors, respectively. ${ }^{13}$ In the most current Children's Oncology Group trial AHEP0731, the surgical guidelines were structured according to PRETEXT. Resection at diagnosis was recommended only for patients with PRETEXT I and II tumors with a $1 \mathrm{~cm}$ radiographic margin from the middle hepatic vein, the retrohepatic inferior vena cava, and the portal bifurcation. Following neoadjuvant chemotherapy, conventional resection is recommended for patients with resolution of major vascular involvement and POSTTEXT I, II, or III group. POSTTEXT refers to the tumor burden after neoadjuvant chemotherapy.

When tumors remain unresectable after chemotherapy, various therapeutic approaches may be employed to facilitate resection. These include consideration of liver transplantation, aggressive nonanatomic resection with vascular reconstruction, and/or interventional embolization depending on the tumor extent and available expertise.

\section{Chemotherapy}

Neoadjuvant chemotherapy allows for delayed resection of the primary mass in some patients with $\mathrm{HB}$; however, certain tumors remain amenable to complete resection only via liver transplantation. Cisplatin is generally considered to be the most active chemotherapy agent in HB treatment and has significantly affected rates of surgical resection and overall survival and is administered alone or in combination with other chemotherapeutic agents (with doxorubicin being the second most common agent utilized). ${ }^{14,15}$ Of note, previous studies have not performed a direct comparison of cisplatin with doxorubicin to determine which is the more active agent in HB treatment. For more advanced HB (including tumors with vascular involvement and those that are PRETEXT IV or metastatic at diagnosis), treatment has been based on multiagent chemotherapy with a cisplatin backbone. For patients with less advanced tumors (eg, tumors without significant vascular involvement), the aim has been to maintain overall survival while decreasing chemotherapy toxicity.

Comparison of different chemotherapeutic regimens has been difficult due to different staging systems, risk stratification schema, and surgical approaches utilized by cooperative groups. Resectability in less advanced tumors is often determined by the expertise, approach, and preference of the treating surgeon. In addition, in North America, the emphasis has been to resect at diagnosis when possible, whereas delayed resection has been advocated in European studies. Event-free survival in patients with HB not resected at diagnosis varies depending on additional prognostic factors as well as the different staging systems and surgical approaches but generally approaches at least $\sim 65 \%-70 \%$ (Table 1). The upcoming international trial will be based on resection at diagnosis when possible and will utilize a singlerisk stratification system. Nonetheless, general retrospective comparisons can be made between the different treatment regimens (Table 1).

Chemotherapy for unresectable HB in North America has historically been based on a cisplatin backbone in combination with 5-fluorouracil and vincristine (C5V) and, most

Table I Resection rates and outcomes in patients with advanced HB

\begin{tabular}{|c|c|c|c|c|c|c|c|c|}
\hline $\begin{array}{l}\text { Time } \\
\text { (location) }\end{array}$ & Study & $\begin{array}{l}\text { Patient } \\
\text { population }\end{array}$ & Chemotherapy & $\begin{array}{l}\text { No of } \\
\text { patients }\end{array}$ & $\begin{array}{l}\text { Not resected } \\
\text { at diagnosis, } n\end{array}$ & $\begin{array}{l}\text { Eligible for } \\
\text { delayed } \\
\text { definitive } \\
\text { resection }^{\mathrm{a}}, \mathrm{n}\end{array}$ & $\begin{array}{l}\text { Resected after } \\
\text { neoadjuvant } \\
\text { chemotherapy, } \\
\text { n (\%) }\end{array}$ & $\mathbf{E F S}^{\mathbf{b}}$ \\
\hline 1989-1992 & INT00989 & Stage III or IV & Cisplatin & 182 & 123 & 104 & $47(45)$ & Stage III: $64 \%$ \\
\hline (North & & & 5-Fluorouracil & & & & & Stage IV: $25 \%$ \\
\hline America) & & & $\begin{array}{l}\text { Vincristine or } \\
\text { cisplatin and } \\
\text { doxorubicin }\end{array}$ & & & & & \\
\hline 1998-2004 & SIOPEL $3 \mathrm{HR}^{16}$ & $\mathrm{HR}^{\mathrm{c}}$ & Cisplatin & 153 & $|5|$ & 127 & 115 (9I) & $65 \%$ \\
\hline (Europe and & & & Carboplatin & & & & & \\
\hline International) & & & Doxorubicin & & & & & \\
\hline $2005-2009$ & SIOPEL $4^{12}$ & $\mathrm{HR}^{\mathrm{c}}$ or tumor & Cisplatin & 62 & 61 & 55 & $52(95)$ & $76 \%$ \\
\hline (Europe and & & rupture & Carboplatin & & & & & \\
\hline International) & & & Doxorubicin & & & & & \\
\hline
\end{tabular}

Notes: ${ }^{a}$ This total excludes patients without data available for analysis. ${ }^{\mathrm{b}}$ Five years for INT0098 and 3 years for SIOPEL studies. ${ }^{\mathrm{CHR}}$ for SIOPEL studies: patients with PRETEXT IV tumor, extrahepatic abdominal disease, vascular invasion, low alpha fetoprotein, or metastatic disease.

Abbreviations: $H B$, hepatoblastoma; EFS, event-free survival; HR, high risk. 
recently, addition of doxorubicin (C5VD) for upfront unresectable patients. In INT0098, 47 patients with unresectable HB ( $45 \%$ of those with tumors not resected at diagnosis and eligible for a delayed resection) underwent complete resection after neoadjuvant chemotherapy. ${ }^{9}$ These patients received in a randomized manner either $\mathrm{C} 5 \mathrm{~V}$ or cisplatin/doxorubicin before the definitive surgery. On close inspection of the INT0098 results, the cisplatin/doxorubicin regimen was actually superior to C5V in terms of disease progression; however, due to excess toxicity, the eventual survival was not statistically different. Given that supportive care measures have improved since then, and with some favorable results from European regimens including doxorubicin, it was decided for the current trial (AHEP0731) that patients with stage III HB should have doxorubicin added to C5V (C5VD). Analysis of patients proceeding to conventional resection or liver transplantation after this new regimen is pending.

Chemotherapy for $\mathrm{HB}$ not resected at diagnosis in Europe has consisted of cisplatin alone for patients with SR HB (localized, PRETEXT I, II, or III tumors) or a combination with carboplatin and doxorubicin for patients with HR HB (PRETEXT IV, metastatic disease, or other HR features on SIOPEL IV). ${ }^{12,13,16}$ In SIOPEL 3SR, 126 patients received either cisplatin monotherapy or cisplatin and doxorubicin with $95 \%$ and $93 \%$ of patients, respectively, able to undergo delayed complete resection. ${ }^{13}$ In SIOPEL 3HR, 150 patients received either cisplatin, carboplatin, and doxorubicin with $56 \%$ able to undergo delayed conventional resection and $21 \%$ proceeding to liver transplantation. ${ }^{16}$ The subsequent SIOPEL study for HR HB (SIOPEL IV) incorporated intensification of the timing of the cisplatin dosing in combination with carboplatin and doxorubicin. ${ }^{12}$ In SIOPEL IV, 74\% of patients were able to undergo delayed complete resection (35\% with liver transplantation and the remaining 65\% with conventional resection).

Taking into account all patients with unresectable HB, there is a subset of patients, as seen in SIOPEL 3SR, for whom less chemotherapy is indicated. ${ }^{13}$ Acute and late toxicities from chemotherapy are significant for patients with HB. The common acute side effects include myelosuppression, malnutrition, febrile neutropenia, and neuropathy. The late effects of chemotherapy for HB include hearing loss, cardiomyopathy, nephrotoxicity, and second neoplasms. Further reduction in chemotherapy exposure with fewer agents and lower cumulative doses will be studied on the upcoming international trial for patients with less advanced $\mathrm{HB}$ not resected at diagnosis.

For the higher risk population with unresectable HB without metastatic disease, the optimal chemotherapeutic approach remains to be determined. For patients with unresectable HB not amenable to conventional resection, liver transplantation is the mainstay for cure. Prolonged chemotherapy administration beyond four cycles in an attempt to proceed with conventional resection has been shown to be ineffective in increasing the ability to proceed to conventional resection and may induce drug resistance. ${ }^{17,18}$

\section{Surgical approaches}

The recommendations for transplant used in the most recent cooperative group studies are: 1) tumor at diagnosis clearly involving all four sections of the liver, especially those with extensive multifocality as judged by MRI or CT angiography and 2) tumor location so close to both main portal vessels at the hilum of the liver and/or all three hepatic veins that it is unlikely that a tumor-free excision plane will be achieved without risking life-threatening hemorrhage. These patients should be identified early in their treatment, and their clinical course and imaging should be followed closely throughout their initial chemotherapy in consultation with a surgeon from a liver specialty center. An occasional patient with an extensively multifocal PRETEXT IV tumor or with tumor thrombosis in the main portal vein might be recommended for primary transplant with minimal preoperative chemotherapy. ${ }^{19}$ The upcoming international trial for HB (Pediatric Hepatic International Tumor Trial) will incorporate risk stratification by the Children's Hepatic Tumors International Collaboration including surgical guidelines based on PRETEXT.

Multifocal PRETEXT IV HB in the absence of any metastatic disease after chemotherapy (POSTTEXT IV + multifocal $/ \mathrm{F}$ - metastases/M) is a clear indication for liver transplantation. Clinicians should resist the temptation to intensify chemotherapy in a vain effort to avoid transplant because of the high likelihood of inducing tumor resistance to chemotherapy. ${ }^{17,18}$ Apparent clearance of tumor nodules from one section of liver after preoperative chemotherapy should not distract from transplant because of the high probability of persistent microscopic viable neoplastic cells despite apparent radiographic clearance. ${ }^{10}$ Children's Oncology Group and SIOPEL recommend transplant in these patients, although there are reports of successful piecemeal resections of such tumors. ${ }^{20}$ The patients with large solitary PRETEXT IV tumors usually receive neoadjuvant chemotherapy, and many of these tumors may "downstage" to POSTTEXT III with clear retraction of the tumor from the anatomic border of one lateral section, allowing for performance of a trisectionectomy. A unifocal POSTTEXT IV tumor without metastatic disease is a clear indication for transplant. 
In a subgroup of patients with PRETEXT II and III tumors, there will be major vascular invasion that does not clear with neoadjuvant chemotherapy. For patients with persistent vascular involvement of all three major hepatic veins, both portal veins, and/or the retrohepatic vena cava (POSTTEXT + venous involvement/V and/or + portal involvement/P), referral for nonconventional liver resection or liver transplantation is recommended. Resection in the face of major venous invasion runs the risk of leaving viable neoplastic tissue behind if the surgeon peels off viable tumor directly from the involved vein. Some have argued in favor of venous resection and reconstruction ("extreme" or "complex" resection) as opposed to transplant in these cases. ${ }^{21}$

Unresponsive or progressive metastatic disease in the face of neoadjuvant chemotherapy is a relative contraindication to transplant because even if the nodules can be surgically resected, microscopic foci of chemoresistant tumor are highly probable. ${ }^{22-24}$ Lung metastases in a patient that seem to respond to chemotherapy, but which do not entirely clear, should be surgically resected. ${ }^{10,25}$ Some have advocated sternotomy and bilateral lung palpation, rather than unilateral wedge resection, although this remains controversial.

\section{Interventional approaches}

Interventional radiology is defined as the use of imaging techniques to guide a minimally invasive procedural intervention. Interventional techniques have long been utilized in pediatrics to obtain central venous access, image the vascular tree, obtain diagnostic tissue via needle biopsy, drain fluid collections, and perform genitourinary and gastroenterology procedures. ${ }^{26,27}$ While the use of interventional techniques to treat adult oncology patients is well established, the translation of these techniques to the pediatric realm continues to evolve. This evolution has been limited by the slow development and approval of new devices for use in children, patient size limitations, and the hesitation to test novel treatment approaches in pediatric patients. ${ }^{28}$ There are numerous interventional radiology modalities available for the treatment of oncology patients, particularly those with liver tumors, with a range of experiences already published in the adult literature. ${ }^{29-32}$ More recently, these interventional approaches have been introduced to the pediatric population as detailed in the following.

Transarterial chemoembolization (TACE) has been widely used in adult hepatocellular carcinoma (HCC) and secondary (colon cancer) liver metastases. It provides the advantage of concentrated chemotherapy administration, increased dwell time, and decreased systemic toxicity. ${ }^{29}$ For hepatic metastases and HCC, TACE can not only be used in combination with radiofrequency ablation (RFA) to treat diffuse disease but may also serve as a neoadjuvant approach to improve the feasibility of local control options such as surgical resection or orthotopic liver transplant. ${ }^{30,31,33}$ TACE and RFA have been trialed in pediatric oncology patients, with patient selection and suitability for intervention extrapolated from the adult experience. Presurgical TACE has been used for pediatric HB in an attempt to reduce tumor volume and aid with surgical intervention. Published series are of various sizes and document a combination of chemotherapeutic agents used. However, nearly all cases demonstrate reduction in tumor size and resultant intratumoral necrosis and decreased intraoperative bleeding. While many publications reported increased ease of surgical resection, not all cases resulted in a complete resection. ${ }^{34-36}$

High-intensity frequency ultrasound is a technique that allows for the focal delivery of high-intensity ultrasound beams directly to the tumor to enhance cell death. Highintensity frequency ultrasound has been trialed with variable success, in combination with TACE, in the pediatric HB patients with upfront unresectable or metastatic tumors. ${ }^{37,38}$

Transarterial radioembolization with yttrium-90, while routinely pursued in the adult community, is relatively new to the pediatric community with experiential case reports limited to the palliative setting. ${ }^{39}$

While RFA has been used extensively in adult liver tumor patients, it has been trialed sparingly in pediatric patients. RFA has the benefit of providing immediate pain relief with a more targeted focus than conventional radiotherapy. ${ }^{40}$ Its use has been reported successfully, albeit for small numbers, in patients with recurrent HB and Wilms tumor liver metastases and in a Phase I/pilot study for all solid tumors in which one patient with fibrolamellar HCC underwent multiple procedures. ${ }^{40-43}$ Postprocedural pain was moderate with prolonged hospitalization more frequently reported. Serious complications were more commonly seen secondary to pulmonary RFA for lung lesions.

\section{Radiation therapy}

Few cases have been reported in the literature incorporating external beam radiation therapy for patients with unresectable HB. Radiation therapy is not indicated during the initial treatment of $\mathrm{HB}$, and its role in recurrent $\mathrm{HB}$ or in $\mathrm{HB}$ not resectable by other means (including patients who are not candidates for liver transplantation) remains to be determined. ${ }^{44-46}$

\section{Stem cell transplantation}

Autologous stem cell transplantation has been explored for patients during upfront treatment as well as in relapse; thus far, it has not been proven beneficial. ${ }^{47,48}$ The specific role of 
stem cell transplantation in rendering HB tumors resectable remains undetermined but is likely to add toxicity in patients who may otherwise be candidates for liver transplantation instead of conventional resection. ${ }^{49}$

\section{Conclusion}

For patients with $\mathrm{HB}$, resection of the primary tumor (and metastatic sites where applicable) is the goal for curative treatment. When tumors are deemed to be unresectable at diagnosis, chemotherapy can be utilized to render most of them resectable. For patients without active metastatic disease but with primary tumors not amenable to conventional resection, liver transplantation is indicated. It stands to reason that for patients with upfront unresectable disease, interventional techniques may serve as a promising option to reduce tumor size, decrease systemic toxicity, and increase ease of tumor resectability. The upcoming international trial for $\mathrm{HB}$ aims to decrease toxicity from chemotherapy for patients with unresectable HB while maintaining good survival.

\section{Disclosure}

The authors report no conflicts of interest in this work.

\section{References}

1. Ries LA, Smith MA, Gurney JG, et al. Cancer Incidence and Survival among Children and Adolescents: United States SEER Program 1975-1995. Bethesda, MD: National Cancer Institute, SEER Program; 1999. NIH Pub No. 99-4649.

2. Darbari A, Sabin KM, Shapiro CN, Schwarz KB. Epidemiology of primary hepatic malignancies in U.S. children. Hepatology. 2003;38(3):560-566.

3. McLaughlin CC, Baptiste MS, Schymura MJ, Nasca PC, Zdeb MS. Maternal and infant birth characteristics and hepatoblastoma. Am J Epidemiol. 2006;163(9):818-828.

4. Linabery AM, Ross JA. Trends in childhood cancer incidence in the U.S. (1992-2004). Cancer. 2008;112(2):416-432.

5. Trobaugh-Lotrario AD, Venkatramani R, Feusner JH. Hepatoblastoma in children with Beckwith-Wiedemann syndrome: does it warrant different treatment? J Pediatr Hematol Oncol. 2014;36(5):369-373.

6. Hirschman BA, Pollock BH, Tomlinson GE. The spectrum of APC mutations in children with hepatoblastoma from familial adenomatous polyposis kindreds. J Pediatr. 2005;147(2):263-266.

7. Spector LG, Feusner JH, Ross JA. Hepatoblastoma and low birth weight. Pediatr Blood Cancer. 2004;43(6):706.

8. Johnson KJ, Williams KS, Ross JA, et al. Parental tobacco and alcohol use and risk of hepatoblastoma in offspring: a report from the children's oncology group. Cancer Epidemiol Biomarkers Prev. 2013;22(10):1837-1843.

9. Ortega JA, Douglass EC, Feusner JH, et al. Randomized comparison of cisplatin/vincristine/fluorouracil and cisplatin/continuous infusion doxorubicin for treatment of pediatric hepatoblastoma: a report from the Children's Cancer Group and the Pediatric Oncology Group. J Clin Oncol. 2000;18(14):2665-2675.

10. Meyers RL, Tiao G, de Ville de Goyet J, Superina R, Aronson DC. Hepatoblastoma state of the art: pre-treatment extent of disease, surgical resection guidelines and the role of liver transplantation. Curr Opin Pediatr. 2014;26(1):29-36.
11. Roebuck DJ, Aronson D, Clapuyt P, et al. 2005 PRETEXT: a revised staging system for primary malignant liver tumours of childhood developed by the SIOPEL group. Pediatr Radiol. 2007;37:123-132.

12. Zsiros J, Brugieres L, Brock P, et al. Dose-dense cisplatin-based chemotherapy and surgery for children with high-risk hepatoblastoma (SIOPEL-4): a prospective, single-arm, feasibility study. Lancet Oncol. 2013;14(9):834-842.

13. Perilongo G, Maibach R, Shafford E, et al. Cisplatin versus cisplatin plus doxorubicin for standard-risk hepatoblastoma. $N$ Engl J Med. 2009;361(17):1662-1670.

14. Black CT, Cangir A, Choroszy M, Andrassy RJ. Marked response to preoperative high-dose cis-platinum in children with unresectable hepatoblastoma. J Pediatr Surg. 1991;26(9):1070-1073.

15. Douglass EC, Green AA, Wrenn E, Champion J, Shipp M, Pratt CB. Effective cisplatin (DDP) based chemotherapy in the treatment of hepatoblastoma. Med Pediatr Oncol. 1985;13(4):187-190.

16. Zsiros J, Maibach R, Shafford E, et al. Successful treatment of childhood high-risk hepatoblastoma with dose-intensive multiagent chemotherapy and surgery: final results of the SIOPEL-3HR study. J Clin Oncol. 2010;28(15):2584-2590.

17. von Schweinitz D, Hecker H, Harms D, et al. Complete resection before development of drug resistance is essential for survival from advanced hepatoblastoma - a report from the German Cooperative Pediatric Liver Tumor Study HB-89. J Pediatr Surg. 1995;30(6):845-852.

18. Warmann SW, Fuchs J. Drug resistance in hepatoblastoma. Curr Pharm Biotechnol. 2007;8(2):93-97.

19. Otte JB. Progress in the surgical treatment of malignant liver tumors in children. Cancer Treat Rev. 2010;36(4):360-371.

20. Lautz TB, Ben-Ami T, Tantemsapya N, Gosiengfiao Y, Superina RA. Successful nontransplant resection of POST-TEXT III and IV hepatoblastoma. Cancer. 2011;117(9):1976-1983.

21. Hemming AW, Mekeel KL, Zendejas I, Kim RD, Sicklick JK, Reed AI. Resection of the liver and inferior vena cava for hepatic malignancy. J Am Coll Surg. 2013;217(1):115-124.

22. Kueht M, Thompson P, Rana A, Cotton R, O’Mahony C, Goss J. Effects of an early referral system on liver transplantation for hepatoblastoma at Texas Children's Hospital. Pediatr Transplant. 2016;20(4): 515-522.

23. Meyers RL, Czauderna P, Otte JB. Surgical treatment of hepatoblastoma. Pediatr Blood Cancer. 2012;59(5):800-808.

24. Ismail $H$, Broniszczak D, Kalicinski $P$, et al. Changing treatment and outcome of children with hepatoblastoma: analysis of a single center experience over the last 20 years. J Pediatr Surg. 2012;47(7): 1331-1339.

25. Gupta AA, Gerstle JT, Ng V, et al. Critical review of controversial issues in the management of advanced pediatric liver tumors. Pediatr Blood Cancer. 2011;56(7):1013-1018.

26. Heran MK, Burrill J. Vascular pediatric interventional radiology. Can Assoc Radiol J. 2012;63(3 suppl):S59-S73.

27. van Sonnenberg E, Wittich GR, Edwards DK, et al. Percutaneous diagnostic and therapeutic interventional radiologic procedures in children: experience in 100 patients. Radiology. 1987;162(3):601-605.

28. Hoffer FA. Interventional oncology: the future. Pediatr Radiol. 2011;41(suppl 1):S201-S206.

29. Albert M, Kiefer MV, Sun W, et al. Chemoembolization of colorectal liver metastases with cisplatin, doxorubicin, mitomycin C, ethiodol, and polyvinyl alcohol. Cancer. 2011;117(2):343-352.

30. Graziadei IW, Sandmueller H, Waldenberger P, et al. Chemoembolization followed by liver transplantation for hepatocellular carcinoma impedes tumor progression while on the waiting list and leads to excellent outcome. Liver Transpl. 2003;9(6):557-563.

31. Morimoto M, Numata K, Kondou M, Nozaki A, Morita S, Tanaka K. Midterm outcomes in patients with intermediate-sized hepatocellular carcinoma: a randomized controlled trial for determining the efficacy of radiofrequency ablation combined with transcatheter arterial chemoembolization. Cancer. 2010;116(23):5452-5460. 
32. Guo W, He X, Li Z, Li Y. Combination of transarterial chemoembolization (TACE) and radiofrequency ablation (RFA) vs. surgical resection (SR) on survival outcome of early hepatocellular carcinoma: a metaanalysis. Hepatogastroenterology. 2015;62(139):710-714.

33. You YT, Changchien CR, Huang JS, Ng KK. Combining systemic chemotherapy with chemoembolization in the treatment of unresectable hepatic metastases from colorectal cancer. Int $J$ Colorectal Dis. 2006;21(1):33-37.

34. Zhang J, Xu F, Chen K, et al. An effective approach for treating unresectable hepatoblastoma in infants and children: pre-operative transcatheter arterial chemoembolization. Oncol Lett. 2013;6(3):850-854.

35. Li JP, Chu JP, Yang JY, Chen W, Wang Y, Huang YH. Preoperative transcatheter selective arterial chemoembolization in treatment of unresectable hepatoblastoma in infants and children. Cardiovasc Intervent Radiol. 2008;31(6):1117-1123.

36. Ohtsuka Y, Matsunaga T, Yoshida H, Kouchi K, Okada T, Ohnuma N. Optimal strategy of preoperative transcatheter arterial chemoembolization for hepatoblastoma. Surg Today. 2004;34(2):127-133.

37. Chen B, Chen J, Luo Q, Guo C. Effective strategy of the combination of high-intensity focused ultrasound and transarterial chemoembolization for improving outcome of unresectable and metastatic hepatoblastoma: a retrospective cohort study. Transl Oncol. 2014;7(6):788-794.

38. Wang S, Yang C, Zhang J, et al. First experience of high-intensity focused ultrasound combined with transcatheter arterial embolization as local control for hepatoblastoma. Hepatology. 2014;59(1):170-177.

39. Hawkins CM, Kukreja K, Geller JI, Schatzman C, Ristagno R. Radioembolisation for treatment of pediatric hepatocellular carcinoma. Pediatr Radiol. 2013;43(7):876-881.

40. Rybak LD, Rosenthal DI, Wittig JC. Chondroblastoma: radiofrequency ablation - alternative to surgical resection in selected cases. Radiology. 2009;251(2):599-604.
41. van Laarhoven S, van Baren R, Tamminga RY, de Jong KP. Radiofrequency ablation in the treatment of liver tumors in children. J Pediatr Surg. 2012;47(3):e7-e12.

42. Hoffer FA, Daw NC, Xiong X, et al. A phase 1/pilot study of radiofrequency ablation for the treatment of recurrent pediatric solid tumors. Cancer. 2009;115(6):1328-1337.

43. Ye J, Shu Q, Li M, Jiang TA. Percutaneous radiofrequency ablation for treatment of hepatoblastoma recurrence. Pediatr Radiol. 2008;38(9):1021-1023.

44. Inaba H, Handgretinger R, Furman W, Hale G, Leung W. Allogeneic graft-versus-hepatoblastoma effect. Pediatr Blood Cancer. 2006;46:501-505

45. Habrand JL, Nehme D, Kalifa C, et al. Is there a place for radiation therapy in the management of hepatoblastomas and hepatocellular carcinomas in children? Int J Radiat Oncol Biol Phys. 1992;23:525-531.

46. Shafer AD, Selinkoff PM. Preoperative irradiation and chemotherapy for initially unresectable hepatoblastoma. J Pediatr Surg. 1977;12: 1001-1007.

47. Karski EE, Dvorak CC, Leung W, et al. Treatment of hepatoblastoma with high-dose chemotherapy and stem cell rescue: the pediatric blood and marrow transplant consortium experience and review of the literature. J Pediatr Hematol Oncol. 2014;36(5): 362-368.

48. Hishiki T, Matsunaga T, Sasaki F, et al. Outcome of hepatoblastomas treated using the Japanese Study Group for pediatric liver tumor (JPLT) protocol-2: report from the JPLT. Pediatr Surg Int. 2011;27(1):1-8

49. Perilongo G, Otte JB. Autologous peripheral blood stem-cell transplantation with a double-conditioning regimen for recurrent hepatoblastoma after liver transplantation - a valid therapeutic option or just too much? Pediatr Transplant. 2009;13(2):148-149.
Hepatic Medicine: Evidence and Research

\section{Publish your work in this journal}

Hepatic Medicine: Evidence and Research is an international, peerreviewed, open access journal covering all aspects of adult and pediatric hepatology in the clinic and laboratory including the following topics: Pathology, pathophysiology of hepatic disease; Investigation and treatment of hepatic disease; Pharmacology of drugs used for the treatment of hepatic disease. Issues of patient safety and quality of care will also be considered. The manuscript management system is completely online and includes a very quick and fair peer-review system, which is all easy to use. Visit http://www.dovepress.com/testimonials.php to read real quotes from published authors. 